\section{GENRE}

en séries

\section{Genre en séries}

Cinéma, télévision, médias

$7 \mid 2018$

Les écrits de la réception : pratiques textuelles des publics médiatiques

\title{
Sandy Montañola et Aurélie Olivesi (dir.), Gender Testing in Sport : Ethics, Cases and Controversies
}

New York, Routledge, 2016, 189 p.

\section{Natacha Lapeyroux}

\section{OpenEdition \\ Journals}

\section{Édition électronique}

URL : http://journals.openedition.org/ges/670

DOI : $10.4000 /$ ges.670

ISSN : 2431-6563

\section{Éditeur}

Presses universitaires de Bordeaux

\section{Référence électronique}

Natacha Lapeyroux, « Sandy Montañola et Aurélie Olivesi (dir.), Gender Testing in Sport: Ethics, Cases and Controversies », Genre en séries [En ligne], 7| 2018, mis en ligne le 01 juin 2018, consulté le 18 février 2021. URL : http://journals.openedition.org/ges/670 ; DOl : https://doi.org/10.4000/ges.670

Ce document a été généré automatiquement le 18 février 2021.

\section{cc) $(1) \odot$}

La revue Genre en séries est mise à disposition selon les termes de la Licence Creative Commons Attribution - Pas d'Utilisation Commerciale - Pas de Modification 4.0 International. 


\title{
Sandy Montañola et Aurélie Olivesi (dir.), Gender Testing in Sport : Ethics, Cases and Controversies
}

\author{
New York, Routledge, 2016, 189 p.
}

\section{Natacha Lapeyroux}

\section{RÉFÉRENCE}

Sandy Montañola et Aurélie Olivesi (dir.), Gender Testing in Sport : Ethics, Cases and Controversies, New York, Routledge, 2016, 189 p.

1 Publié en 2016 aux éditions Routledge sous la direction de Sandy Montañola et Aurélie Olivesi ${ }^{1}$, l'ouvrage Gender Testing in Sport: Ethics, Cases and Controversies fait suite à un travail collectif de recherche mené entre 2011 et 2015 ainsi qu'au colloque «'L'affaire Semenya' : approches disciplinaires » qui s'est déroulé à l'Université Lyon 1 en $2013^{2}$. Il traite de la controverse autour de "l'affaire Caster Semenya » rendue publique en 2009 lorsque l'IAAF ${ }^{3}$ a demandé à l'athlète sud-africaine de se soumettre à un test de féminité ${ }^{4}$ à la suite de sa victoire à l'épreuve du 800 mètres des femmes aux Championnats du Monde de Berlin. Suspectée de dopage, d'être hermaphrodite ou encore d'être un homme, l'athlète est suspendue de compétition durant la durée des tests. Le "problème public $»^{5}$ consiste alors à déterminer l'identité sexuelle de Caster Semenya.

2 La première partie de l'ouvrage (chapitres 1 à 4) interroge la manière dont les institutions sportives, juridiques et médicales construisent les normes corporelles sexuelles autour d'une conception binaire du système sexe/genre et du principe de justice et d'équité dans le sport. Comme le note Philippe Liotard (chapitre 1), Caster Semenya apparaît comme un être hors-norme dans un contexte sportif fondé sur "l'économie des différences corporelles » (p.13), qui opère une ségrégation en fonction des différentes catégories anatomiques (d'âge, de sexe, de poids) et légitime la 
bipartition homme/femme au sein des compétitions sportives. Durant cette compétition, l'athlète âgée de 18 ans, noire et pauvre, fut jugée « trop masculine », trop "jeune ", trop " performante" et suspectée d'homosexualité. Elle était en d'autres termes perçue comme étant trop transgressive par rapport aux normes de féminité stéréotypées pour être une femme, semant du trouble dans le genre ${ }^{6}$. Anaïs Bohuon et Eva Rodriguez (chapitre 2), grâce à un retour sur l'histoire des tests de féminité et de la lutte contre le dopage, montrent qu'imposer un test de féminité à Caster Semenya apparaît alors légitime dans cette affaire afin de remettre de l'ordre dans les catégories de genre en s'appuyant sur le principe de justice qui se trouve au fondement des valeurs sportives. L'avantage conféré à l'athlète en raison d'un taux de testostérone plus élevé que celui autorisé pour les femmes lors des compétitions va conduire les institutions sportives à réguler le taux hormonal des athlètes intersexuées à partir de 2011. Cette mesure atteste selon les auteures d'une confusion entre dopage, masculinité des athlètes et triche construite autour de la dichotomie naturel/artificiel. Pourtant, Silvia Comporesi et Paolo Maugeri (chapitre 3) le soulignent, si les standards médicaux et éthiques soutiennent la régulation de l'hyperandrogénisme, il existe d'autres variations génétiques procurant un avantage physique aux athlètes et qui ne sont pas sanctionnées par les autorités sportives. Cette régulation du taux de testostérone des athlètes intersexuées semble alors avoir pour fonction implicite de perpétuer les normes sociales binaires en matière d'identité sexuelle et de genre. Selon Laurence Brunet et Muriel Salle (chapitre 4), il apparaît donc moral d'imposer juridiquement une hormonothérapie aux athlètes intersexuées alors que l'éthique médicale doit en principe respecter l'autonomie des patient.e.s. Les corps médical et juridique, travaillant de concert mais avec des perspectives différentes ${ }^{7}$, modèlent et font évoluer au cours de l'histoire la perception et la définition des personnes intersexuées, tout en disciplinant leur corps.

3 À partir d'un corpus transnational, la seconde partie (chapitres 5 à 9) questionne les représentations médiatiques de "l'affaire Caster Semenya " - qui ont fait de l'athlète un objet de discours en raison de sa déviance par rapport à la norme - ainsi que les régimes discursifs véhiculés par les différents protagonistes ou au contraire les silences concernant l'identité de l'athlète. Aurélie Olivesi (chapitre 5) appréhende la controverse autour de l'athlète sud-africaine comme un moment discursif ${ }^{8}$ (Moirand, 2007) qui a laissé une trace dans la mémoire collective. Après 2009, il sera toujours fait référence à Caster Semenya lorsqu'il est question d'une athlète dont la féminité est questionnée. De plus, si l'affaire soulève des interrogations sur ce que sont une compétition juste, le système sexe/genre ou l'identité sexuelle, la polémique mène néanmoins à une aporie. En effet, les discours des autorités sportives, des journalistes, des médecins, des compétiteurs (etc.), sont formulés à la négative (ne pas tricher, ne pas excéder certaines normes physiques, ne pas partager les résultats médicaux) ce qui ne permet pas de faire émerger et de stabiliser de nouvelles normes. Seuls les discours sud-africains sont affirmatifs et dénoncent l'humiliation et l'injustice subies par l'athlète dans un contexte postcolonial. Par ailleurs, l'identité de Caster Semenya est présentée comme étant problématique selon les médias et les documents officiels de l'IAAF qui envisagent «le genre » en tant que prolongement naturel du sexe, et le conçoivent donc comme devant être visuellement évident (Fabien Rose, chapitre 6). L'hypothèse intersexuée vient alors donner du sens à un genre inintelligible selon les définitions occidentales et essentialisantes de l'identité féminine. Sandy Montañola (chapitre 7), en modélisant les luttes discursives menées par les différents 
protagonistes concernant les normes de féminité, met en lumière une opposition entre la voix africaine (qui dénonce le sexisme, le racisme dans un contexte post-apartheid) et les institutions sportives internationales valorisant les valeurs de justice et d'équité et les normes de féminité occidentales. Les médecins sont convoqués par les médias pour délivrer "la vérité", alors que les chercheur.e.s en sciences sociales et en bioéthique sont quant à eux sollicités pour donner leurs éclairages au sujet de "l'affaire». Dans ce contexte, Elaine Salo (chapitre 8) analyse le silence de Caster Semenya comme le moyen pour l'athlète de faire preuve de capacité d'agir face aux différents régimes discursifs disciplinaires circulant dans la sphère publique au sujet de son corps qui peuvent avoir des effets matériels (suspension de la compétition ou au contraire autorisation de concourir, régulation du taux de testostérone etc.). Enfin, selon John Sloop (chapitre 9), le silence des autorités sportives dans les médias au sujet du résultat du test de féminité et de la décision d'autoriser Caster Semenya à réintégrer la compétition en 2010 donne lieu à des évolutions progressistes tout en réaffirmant les représentations binaires du système sexe/genre. En effet, si le silence autour de l'identité de Caster Semenya signifie qu'elle peut continuer à concourir au sein des compétitions "féminines", l'athlète doit pour autant conformer son identité à la définition juridique de ce qu'est une femme biologique selon les instances sportives'.

4 L'approche pluridisciplinaire des chercheur.e.s en sciences humaines et sociales ainsi que l'origine internationale des contributeurs et contributrices ${ }^{10}$ de cet ouvrage sont particulièrement pertinentes pour aborder la complexité de «l'affaire Caster Semenya " qui a connu un retentissement médiatique international et interroge les institutions sportives mondiales sur la difficulté à donner une définition stable de ce qu'est une femme biologique « authentique». En effet, l'ouvrage se situe au carrefour de la sociologie du sport, du genre, des sciences de l'information et de la communication, de la bioéthique et du domaine juridique et rend compte de la façon dont le système sexe/genre est produit dans le domaine sportif par des «technologies de genre ${ }^{11}$ ) - les autorités sportives, les médecins, les juristes et les médias - qui construisent et véhiculent des normes sociales binaires, naturalisantes, essentialisantes et hétéronormatives relatives aux identités sexuelles et aux genres. Elles reproduisent par ce biais la hiérarchie entre les sexes (les hommes sont jugés plus performants que les femmes). L'étude de « l'affaire Caster Semenya » révèle que les femmes réalisant des résultats sportifs trop spectaculaires et dont l'apparence physique est en inadéquation avec les canons de beauté stéréotypés sont suspectées d'être dopées, hermaphrodites ou d'être des hommes. Comme le note Silvia Camporesi dans la postface, cette affaire s'inscrit ainsi dans une longue histoire de régulation des corps féminins ("Afterword $»)^{12}$. Enfin, dans le but de rendre compte des discriminations qu'a subies l'athlète en raison de son identité multiple (femme intersexuée, noire, sud-africaine, lesbienne et issue d'un milieu populaire), les auteur.e.s ont analysé «l'affaire Caster Semenya " en mobilisant une approche intersectionnelle au prisme des rapports sociaux de sexe, de race, de classe, de genre, de sexualité, d'âge et dans une perspective postcoloniale.

5 En août 2015, le TAS ${ }^{13}$, saisi par l'athlète indienne Dutee Chand, a suspendu durant deux années la décision rendue par l'IAAF de réguler l'hyperandrogénisme. Pour la première fois dans l'histoire du sport, les athlètes intersexuées ont été autorisées à concourir sans limitation de leur taux de testostérone sept années après le début de la controverse autour de l'athlète sud-africaine. Le TAS a finalement statué en faveur de la régulation du taux de testostérone des athlètes intersexuées à compter du 
$1^{\text {er }}$ novembre 2018 pour les courses allant du 400 mètres au 1500 mètres, à savoir celles dans lesquelles Caster Semenya concourt. La Fédération d'athlétisme sud-africaine a en retour décidé de contester cette décision jugée "raciste», "sexiste» et «homophobe».

6 Il pourrait alors être intéressant de prolonger cette étude en interrogeant les institutions sportives à ce sujet, en analysant les représentations des épreuves dans lesquelles Caster Semenya concourt entre 2016 et 2018, ainsi que la suite de la controverse.

\section{NOTES}

1. Sandy Montañola, et Aurélie Olivesi sont maîtresses de conférences en sciences de l'information et de la communication, respectivement à l'Université de Rennes 1 et à l'Université Claude Bernard Lyon 1.

2. Journées d'étude organisées par Sandy Montañola (CRAPE, Rennes 1), Aurélie Olivesi (ELICO, Lyon 1) et Arnaud Richard (PRAXILING, Montpellier 3).

3. International Association of Athletics Federations.

4. Anaïs Bohuon (2012), Les Tests de féminité, Donnemarie-Dontilly, iXe.

5. Daniel Cefaï (1996), « La construction des problèmes publics. Définitions de situations dans des arènes publiques ", Réseaux, vol. 14, $\mathrm{n}^{\circ}$ 75, p. 43-66.

6. Judith Butler (2005), Trouble dans le genre. Pour un féminisme de la subversion, Paris, La Découverte.

7. La loi commence à prendre en compte l'auto-détermination alors que la médecine n'a jamais remis en question les assignations traditionnelles.

8. Sophie Moirand (2007), Les Discours de la presse quotidienne : observer, analyser, comprendre, Paris, Presses universitaires de France.

9. Caster Semenya doit suivre une hormonothérapie pour réguler son taux de testostérone ce qui a pour conséquence d'amoindrir ses performances physiques.

10. France, Italie, États-Unis, Canada, Afrique du Sud.

11. Teresa De Lauretis (2007), Théorie queer et cultures populaires. De Foucault à Cronenberg, traduit par Marie-Hélène Bourcier, Paris, Éditions La Dispute.

12. Les corps "féminins" ont toujours été l'objet de régulations contraignantes. Celles-ci évoluent au cours de l'histoire en fonction des contextes sociaux (interdiction de porter un pantalon, d'avorter, de pratiquer du sport etc.).

13. Le Tribunal Arbitral du Sport. 


\section{AUTEURS}

\section{NATACHA LAPEYROUX}

Natacha Lapeyroux est doctorante en sciences de l'information et de la communication à l'Université Sorbonne Nouvelle - Paris 3 et actuellement ATER à l'Université de Lorraine. Elle effectue une thèse sous la direction d'Éric Maigret intitulée «L'identité féminine à l'épreuve du sport. Représentations télévisuelles des sportives de haut-niveau ». Ses recherches se situent au croisement de la sociologie des médias, du genre et du sport. 